\title{
Symposium on fractures of the thoracic and the lumbar spine
}

\author{
Cl. Schwartz ${ }^{1}$ and J.P. Steib ${ }^{2}$ \\ ' Service d'Orthopédie-Traumatologie, Centre Hospitalier Louis-Pasteur, F-68021 Colmar Cedex, France \\ ${ }^{2}$ Hôpital Chirurgical Orthopédique Stéphanie, F-67026 Strasbourg Cedex
}

The Board of GECO has entrusted us with a Traumatology subject this year "Fractures of the Thoracic and Lumbar Spine".

We all have to deal with these fractures at some time or another, even without a special interest in spinal surgery. That we all have to treat such patients is shown by our collecting 317 fractures, all treated by GECO members, or in units in which they work. It therefore seemed important to establish an audit of the past 10 years to ascertain the wisdom on operating on more and more of these fractures (the number has doubled in 10 years!).

The subject is limited to fresh fractures, treated initially by GECO members, but without other discriminations about age, severity or treatment. This symposium should become neither an annoying presentation with statistic and computers, nor kindle a partisan conflict among superspecialists, where the simple facts become blurred and inintelligible. In the symposium we aim to interest everyone, including those who practice this kind of surgery regularly. Their contribution is mandatory because of the rapid changes in this field.

In the first part we are going to refresh our memories with the anatomy, bio-mechanics and radiology. We use Francis Denis classification, which is the most used and most logical. This will allow us to find a common language. Thereafter we shall deal with the neurology associated with these fractures and then we shall deal with the orthopaedic and functional treatment. Finally we shall analyse the GECO series.

J.M. Balliet has provided an excel- lent and reliable statistical analysis. Only cases with complete data and a minimum follow-up of 6 months have been selected. Many cases were therefore discarded because of inadequate data. The results need to be carefully scrutinised, acknowledging all concomitant factors, so that percentages and numbers maintain real significance. Interpretation has been most cautious.

The second part of our symposium investigates surgical treatment, be it posterior with the Roy-Camille, CotrelDubousset or Hartshill devices, or be it anterior or even double. Here we will benefit from the great experience of the invited participants. After having reviewed the surgical treatments in this GECO series we shall look at the results and learn what is of value in the common experience, particularly what conclusions can be drawn for the future. 